\title{
Expression profile of Caenorhabditis elegans mutant for the Werner syndrome gene ortholog reveals the impact of vitamin C on development to increase life span
}

\author{
Alexandra Dallaire, Sophie Proulx, Martin J Simard and Michel Lebel
}

\begin{abstract}
Background: Werner Syndrome (WS) is a rare disorder characterized by the premature onset of a number of age-related diseases. The gene responsible for WS encodes a DNA helicase/exonuclease protein believed to affect different aspects of transcription, replication, and DNA repair. Caenorhabditis elegans (C. elegans) with a nonfunctional wrn-1 DNA helicase ortholog also exhibits a shorter life span, which can be rescued by vitamin C. In this study, we analyzed the impact of a mutation in the wrn-1 gene and the dietary supplementation of vitamin $\mathrm{C}$ on the global mRNA expression of the whole C. elegans by the RNA-seq technology.

Results: Vitamin C increased the mean life span of the wrn-1 (gk99) mutant and the N2 wild type strains at $25^{\circ} \mathrm{C}$. However, the alteration of gene expression by vitamin C is different between wrn-1(gk99) and wild type strains. We observed alteration in the expression of 1522 genes in wrn-1(gk99) worms compared to wild type animals. Such genes significantly affected the metabolism of lipid, cellular ketone, organic acid, and carboxylic acids. Vitamin C, in return, altered the expression of genes in wrn-1(gk99) worms involved in locomotion and anatomical structure development. Proteolysis was the only biological process significantly affected by vitamin C in wild type worms.

Conclusions: Expression profiling of wrn-1 ( $\mathrm{kg99}$ ) worms revealed a very different response to the addition of vitamin C compared to wild type worms. Finally, vitamin C extended the life span of wrn-1(gk99) animals by altering biological processes involved mainly in locomotion and anatomical structure development.
\end{abstract}

\section{Background}

WS is a human autosomal recessive disorder characterized by genomic instability and the premature onset of a number of age-related diseases [1-4]. The defective enzyme responsible for WS possesses a $3^{\prime}-5^{\prime}$ exonuclease activity in addition to a $3^{\prime}-5^{\prime}$ helicase activity $[5,6]$ and is involved in DNA repair, replication, transcription, and telomere maintenance [7-11]. We previously generated a mouse model with a deletion in the helicase domain of the murine WRN homologue [12] that recapitulates most of the WS phenotypes, including an abnormal hyaluronic acid excretion, higher reactive oxygen species (ROS) levels, dyslipidemia, increased genomic instability,

\footnotetext{
* Correspondence: michel.lebel@crhdq.ulaval.ca

Centre de Recherche sur le Cancer de l'Université Laval, Hôpital Hôtel-Dieu de Québec (CHU de Québec Research Center), 9 McMahon Sreet, Québec City G1R 2 J6, Canada
}

and cancer incidence [13,14]. Overall, such mutant mice have a $10-15 \%$ decreased of their mean life span $[15,16]$. Interestingly, the treatment of $\mathrm{Wrn}$ mutant mice with vitamin $C$ reverted the life span of such animals to the normal wild type phenotype [16].

The WRN protein is a member of the RecQ family of DNA helicases [3]. It is highly conserved across species including in invertebrates such as the small worm Caenorhabditis elegans (C. elegans). Interestingly, the exonuclease and the DNA helicase domains homologous to the human WRN protein are encoded by two different genes in C. elegans [17]. The C. elegans wrn-1 gene codes for the ATPdependent $3^{\prime}-5^{\prime}$ DNA helicase capable of unwinding a variety of DNA structures [18]. Notably, it has been shown that the RNAi knockdown of the C. elegans wrn1 gene shortens the life span, increases sensitivity to DNA damage, and accelerates aging phenotypes [17]. 
Similarly, a $C$. elegans strain lacking the expression of the $w r n-1$ helicase protein (wrn-1 (gk99)) also exhibit a shorter life span when grown at $25^{\circ} \mathrm{C}$ [19]. Interestingly, supplementation of vitamin $\mathrm{C}$ normalizes the median life span of the wrn-1 (gk99) mutant worms [19] as seen in mice lacking part of the helicase domain of the Wrn protein [16]. Thus, the wrn-1 ( $k$ k99) mutant worm model gives us the opportunity to examine the global mRNA expression of a whole animal upon vitamin $\mathrm{C}$ treatment.

In this study, we analyzed the global mRNA expression of wild type and wrn-1 ( $k$ k99) mutant C. elegans in the presence or absence of vitamin C by RNA-seq. RNA-seq has been shown to be quantitatively accurate over a larger range of expression levels than previous methods, such as microarrays [20-24]. Our results indicate that wrn-1 mutant animals showed significant changes in biological processes affecting several carbon structures (including lipid metabolisms) and oxidation-reduction reactions. Although supplementation of vitamin $\mathrm{C}$ significantly increased the life span of both wild type and wrn-1 mutant worms, no gene was similarly affected by vitamin $C$ in these strains. Vitamin C altered the expression of genes in wrn-1 ( $g$ k99) worms involved in locomotion and anatomical structure development. Proteolysis was the only biological process significantly affected by vitamin $C$ in wild type worms. These results indicate that the expression profiling of $w r n-1(g k 99)$ worms revealed a very different response to the addition of vitamin $\mathrm{C}$ compared to wild type worms.

\section{Results}

Vitamin C increases the life span of both wrn-1(gk99) and wild type $C$. elegans strains

To assess the impact of vitamin $C$ on longevity, we measured the life span of wrn-1 $(g k 99)$ and wild type C. elegans strains grown at $25^{\circ} \mathrm{C}$ in the absence or presence of $10 \mathrm{mM}$ ascorbate (vitamin C) as described before [19,25]. As shown in Figure 1, the median life span of vitamin C treated wrn-1 $($ k 99$)$ worms was significantly increased by $26 \%$ (7.0 days versus 8.8 days) compared to untreated wrn1 ( $\mathrm{k}$ k9) animals (log-rank test: $\left.P=1.83 \times 10^{-6}\right)$. These results replicate previous data obtained with these strains [19]. Interestingly, the median life span of vitamin C treated wrn-1 ( $k$ k99) worms was close to the median life span of wild type worms (Figure 1). The median life span of vitamin $C$ treated wild type worms was significantly increased by $22 \%$ (9.0 days versus 11.0 days) compared to the untreated wild type animals (log-rank test: $P=2.07 \times$ $10^{-5}$ ). These results indicate that vitamin $C$ increased the life span of both strains by more than $20 \%$ at $25^{\circ} \mathrm{C}$.

Transcriptome characterization of the wrn-1(gk99) animals compared to the wild type strain

To gain insight into the rescuing effect of vitamin $\mathrm{C}$ on the life span of wrn-1(gk99) worms, we first established

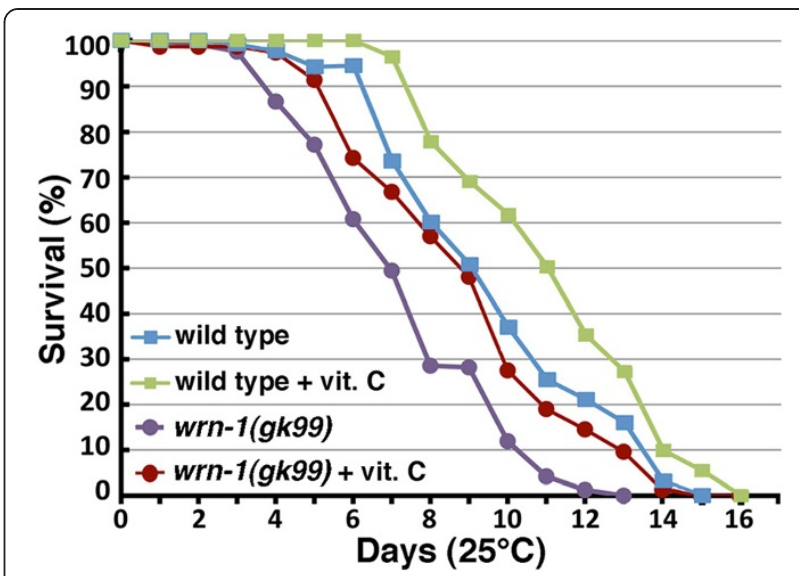

Figure 1 Impact of vitamin $C$ on the life span of wild type and wrn-1 mutant $\boldsymbol{C}$. elegans strains. The difference in longevity between vitamin C treated wrn-1 (gk99) and untreated wrn-1 (gk99) C. elegans strain grown at $25^{\circ} \mathrm{C}$ is significant (log rank test; $P=1.83 \times$ $10^{-6}$ ). The difference in longevity between vitamin $C$ treated wild type (N2) and untreated wild type (N2) C. elegans strain grown at $25^{\circ} \mathrm{C}$ is significant (log rank test; $P=2.07 \times 10^{-5}$ ). All experiments were performed four to six times with 20 to 30 worms per genotype.

$P$-values were obtained using the log-rank test method.

the global expression profile of untreated wild type and mutant worms at the L4 stage by RNA-seq. By carefully monitored physiological developmental cues (such as vulva and gonad development), we noticed a three hours developmental delay in wrn-1 ( $k$ k99) mutants compared to wild type animals. Therefore, mRNA was extracted based on the biological age (i.e. the exact developmental stage) and not on the chronological age of the animals for the RNA-seq analyses. Table 1 contains the number of raw reads for each biological replicate (including the vitamin C-treated worms). The normalized counts for

\section{Table $1 \mathrm{C}$. elegans genotypes used in this study}

\begin{tabular}{lc}
\hline Sample name & Number of reads \\
\hline N2-1 & $18,288,816$ \\
N2-2 & $18,694,683$ \\
N2-3 & $23,406,553$ \\
N2-1 + vitC & $19,647,258$ \\
N2-2 + vitC & $19,580,669$ \\
N2-3 + vitC & $24,862,436$ \\
Wrn-1-1 & $37,999,237$ \\
Wrn-1-2 & $26,388,834$ \\
Wrn-1-3 & $21,700,537$ \\
Wrn-1-1 + vitC & $29,673,436$ \\
wrn-1-2 + vitC & $15,031,408$ \\
wrn-1-3 + vitC & $25,050,297$
\end{tabular}

vitC $=10 \mathrm{mM}$ of vitamin C (ascorbate) in media. 
each biological replicate are shown in the Additional file 1: Table S1 for all the C. elegans genes. A preliminary list of differentially expressed genes was generated by keeping the genes that showed a two-fold difference or more between wild type $\mathrm{N} 2$ and wrn-1 $(g k 99)$ worms with an adjusted $P$-value $<0.01$. This first list contained 2528 genes (data not shown). We randomly chose 16 genes representing a range of base mean number from 4 to 3500 reads in both or in either N2 or wrn-1 mutant animals based on the RNA-seq normalized data (list of genes in Table 2) to determine the minimum of reads required for a gene to be detected by RT-PCR in our analyses. We thus designed 16 pairs of primers to validate the differential expression of these genes by quantitative RT-PCR. The tubulin encoding gene $(t b a-1)$ was included as a control (no difference in $t b a-1$ expression between both strains). PolyA + RNA was thus extracted from three plates of wild type and from three plates of wrn-1(gk99) worms (each plate representing one biological replicate of worms at the L4 stage). The RT-PCR results are shown in Table 2 and the sequences of the primers are provided in the Additional file 2: Table S2. Every RT-PCR product was examined on a $2 \%$ agarose gel at the end of each run. Figure 2 shows examples of such gels for ten of these genes. A PCR product was detected only in samples that showed more than 145 reads by RNA-seq (Additional file 1: Table S1). For example, no band corresponding to nlp-32 mRNA could be detected with the mRNA samples and no Ct could be detected by quantitative RT-PCR. The mean number of reads is below 134 for $n l p-32$ mRNA in both the wild type and the wrn-1(gk99) worms (Table 2). Similarly, no arrd-24 mRNA could be detected from wild type or $w r n-1(g k 99)$ worms mRNA pools even after 40 cycles of PCR (Figure 2). The normalized counts for the arrd-24 gene were below 32 in both genotypes for all biological replicates (Table 2). No Ct could be detected by quantitative RT-PCR. In contrast, a band for the ugt-33 gene was detected with mRNA from the wild type worms only (no band was detected for the wrn-1(gk99) worms) (Figure 2). The mean number of reads was lower than 145 for ugt-33 in the wrn-1(gk99) worms but higher than 145 in the wild type worms (Table 2). Finally, Table 2 indicated expression differences for pes-8, cyp-34a9, cyp33e2, sodh-1, ugt-33, ech-9, nas-3, gpx-3, acs-2, acs-14, asah-1, lys-8, Y46G5.20, and daf-9 genes between the wild type and the wrn-1 $(g k 99)$ strains by RT-PCR analyses and such results confirmed the RNA-seq data. A mean number of reads greater than 145 was found in at least one of the genotypes following the RNA-seq analysis for these genes (Table 2). Based on such results, we generated a final list of genes showing at least a two-fold difference in expression between wild type and mutant worms, an adjusted $P$-value $<0.01$, and a mean number of reads $>145$ in at least one of the worm strain. These criteria were applied to each comparison hereafter in

Table 2 Quantitative RT-PCR compared to RNA-seq data for 16 genes $^{\text {a }}$

\begin{tabular}{|c|c|c|c|c|c|c|}
\hline \multicolumn{4}{|l|}{ RNA-seq } & \multicolumn{3}{|c|}{ RT-PCR } \\
\hline Gene & Base mean in N2 & Base mean in wrn-1 & $\begin{array}{l}\text { Fold difference } \\
\text {. }\end{array}$ & $\Delta \mathrm{Ct}$ & Fold difference & P-value \\
\hline sodh-1 & 465 & 5021 & +10.79 & 3.02 & +8.09 & $<0.001$ \\
\hline $\operatorname{arrd}-24$ & 4 & 32 & +7.80 & none & N/A & N/A \\
\hline$g p x-3$ & 24 & 146 & +6.21 & 1.07 & +3.24 & 0.0012 \\
\hline nas-3 & 49 & 220 & +4.51 & 1.78 & +3.43 & $<0.001$ \\
\hline daf-9 & 168 & 605 & +3.60 & 1.48 & +2.78 & $<0.001$ \\
\hline asah-1 & 393 & 1200 & +3.06 & 0.72 & +1.65 & 0.0008 \\
\hline acs-2 & 594 & 1277 & +3.30 & 1.80 & +3.48 & $<0.001$ \\
\hline $\operatorname{acs}-14$ & 763 & 2289 & +3.00 & 0.52 & +1.43 & 0.0307 \\
\hline ech-9 & 155 & 449 & +2.89 & 1.94 & +3.84 & 0.0006 \\
\hline Y46G5.20 & 1182 & 381 & -3.10 & -2.05 & -4.14 & 0.0001 \\
\hline сур-33е2 & 1708 & 548 & -3.12 & -2.70 & -6.52 & $<0.001$ \\
\hline cyp-34a9 & 987 & 289 & -3.41 & -2.05 & -4.15 & $<0.001$ \\
\hline pes-8 & 256 & 66 & -3.84 & -2.06 & -4.16 & 0.0204 \\
\hline ugt-33 & 269 & 69 & -3.90 & -1.67 & -3.18 & 0.0008 \\
\hline lys -8 & 3405 & 155 & -21.93 & -4.45 & -21.87 & $<0.001$ \\
\hline$n / p-32$ & 135 & 5 & -28.69 & none & N/A & $\mathrm{N} \backslash \mathrm{A}$ \\
\hline$t b a-1^{b}$ & 12597 & 10618 & -1.19 & -0.12 & -1.09 & 0.7083 \\
\hline
\end{tabular}

${ }^{\mathrm{a}} \mathrm{RT}-\mathrm{PCR}$ were performed in triplicate and all values represent the means.

${ }^{\mathrm{b}}$ The $t$ ba-1 gene was used as a control (no difference of expression between N2 and wrn-1 mutants). 


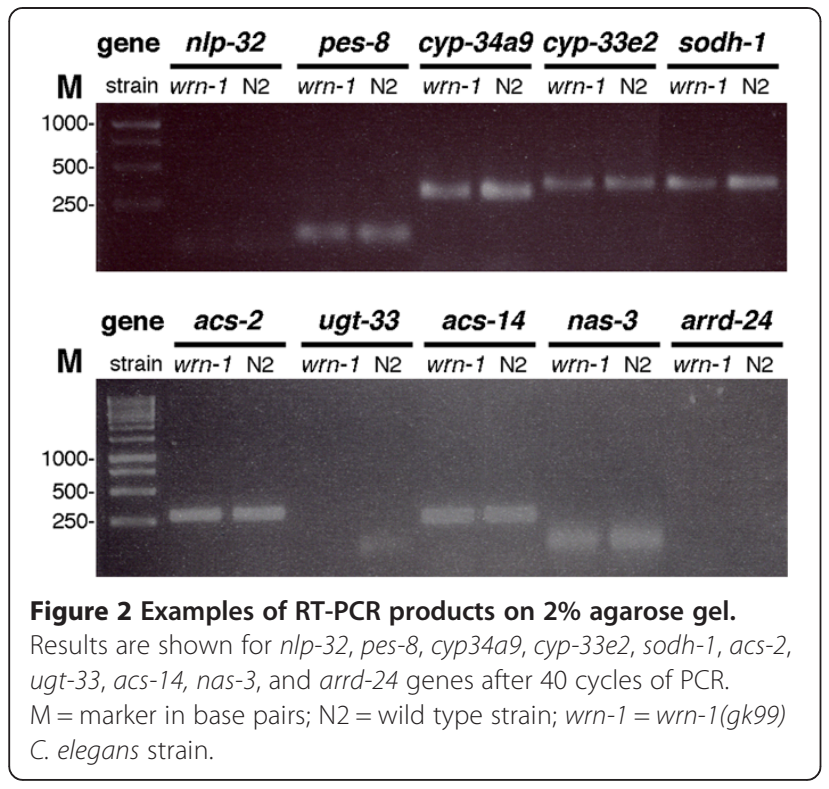

this report. Additional file 3: Table S3 gives a final list of 1522 genes differentially expressed between wild type and wrn-1 $(g k 99)$ worms. There were 907 down regulated and 615 up regulated genes respectively (by at least twofold) in the wrn-1(gk99) worms compared to the wild type worms.

The DAVID classification tool was used to classify differentially expressed genes by their functions in specific biological processes. Categorization of the genes in our list (Additional file 3: Table S3) revealed ten significantly up regulated biological processes (with a Benjamini $P$ value $<0.05)$ in the $w r n-1(g k 99)$ worms compared to wild type worms (Table 3). Major biological processes that were up regulated in wrn-1( $g k 99)$ worms included oxidationreduction processes, lipid, organic acid, cellular ketone, and carbohydrate metabolic processes (Table 3). There was no significant down regulated biological processes (with a Benjamini $P$-value $<0.05$ ).

\section{Transcriptome characterization of wrn-1(gk99) and wild type strains treated with vitamin $C$}

We compared the global mRNA expression of synchronized untreated worms with synchronized worms grown with vitamin $\mathrm{C}$ until the L4 stage. Lists of genes differentially expressed were generated by requiring that the absolute value of the fold change be higher than two, that the mean number of reads for each gene be higher than 145 for at least one of the genotype, and the adjusted $P$ value be lower than 0.01 . When we compared the global expression of untreated wrn-1( $\mathrm{k} \mathrm{k} 99)$ worms with vitamin C treated wrn-1(gk99) worms, we found 311 genes to be differentially expressed (139 down and 172 up regulated genes, respectively) between both populations (Additional file 4: Table S4). DAVID analysis on this list of genes revealed enrichments for two main down regulated biological processes (Table 4). The main affected processes included genes involved in locomotion and anatomical structure development. No significant biological processes were up regulated (with a Benjamini P-value $<0.05$ ). When we compared the global expression of untreated wild type worms with vitamin $C$ treated wild type worms, we found 296 genes to be differentially expressed (193 down and 103 up regulated genes, respectively) between both populations (Additional file 5: Table S5). DAVID analysis on this list of genes revealed a significant down regulation of only one set of genes involved in proteolysis (Table 4).

Table 3 Biological processes altered in wrn-1(gk99) C. elegans strain compared to the wild type N2 strain

\begin{tabular}{|c|c|c|}
\hline \multicolumn{3}{|l|}{ Up in wrn-1(gk99) C. elegans } \\
\hline Category & $\begin{array}{c}\text { Benjamini } \\
P \text {-value }\end{array}$ & Genes \\
\hline Organic acid metabolic process & $1.34 \mathrm{E}-04$ & $\begin{array}{l}\text { R09B5.6, F08A8.3, F56H11.4, F08A8.1, C12C8.2, F08A8.2, F28F8.2, M02D8.4, W08D2.4, C48B4.1, } \\
\text { C44E4.3, C05E4.9, T21F4.1, T09B4.8, F01G10.3, K07E1.1, Y73F4A.2, F21 F8.4, D1025.2, W09B6.1, } \\
\text { F59B8.2, ZC416.6, ZK1290.12, F13D12.4, K10H10.2, ZK829.4, R08E5.2, R11A5.4 }\end{array}$ \\
\hline Cellular ketone metabolic process & $1.78 \mathrm{E}-04$ & $\begin{array}{l}\text { R09B5.6, F08A8.3, F56H11.4, F08A8.1, C12C8.2, F08A8.2, F28F8.2, M02D8.4, W08D2.4, C48B4.1, } \\
\text { C44E4.3, C05E4.9, T21F4.1, T09B4.8, F01G10.3, K07E1.1, Y73F4A.2, F21F8.4, D1025.2, W09B6.1, } \\
\text { F59B8.2, ZC416.6, ZK1290.12, F13D12.4, K10H10.2, ZK829.4, R08E5.2, R11A5.4 }\end{array}$ \\
\hline Lipid metabolic process & $2.52 \mathrm{E}-04$ & $\begin{array}{l}\text { R09B5.6, F08A8.3, F08A8.1, F56H11.4, F28F8.2, F08A8.2, K11D2.2, W08D2.4, Y52B11A.8, F09C8.1, } \\
\text { C48B4.1, ZK6.7, T19H12.9, T05E7.1, F59F4.4, F46G10.4, F01G10.3, C40H1.7, H23N18.1, Y37E11AR.5, } \\
\text { F11E6.5, T12B3.3, Y54G2A.45, F22E10.5, F21F8.4, F35C11.5, T19H12.10, W09B6.1, F28H7.3, ZC416.6, } \\
\text { Y49E10.18, C31H5.6, C33C12.3, F54D11.1, W04C9.1 }\end{array}$ \\
\hline Oxidation-reduction & 0.0072 & $\begin{array}{l}\text { T10B9.7, Y39A1A.19, T13C5.1, F08A8.2, C47A10.5, T08B1.3, F54D8.3, C48B4.1, K08C7.5, R02D3.1, } \\
\text { C26F1.2, C02C2.1, F55B11.1, C11E4.2, R07B7.5, K06A4.5, F08F3.7, F36A2.3, R11D1.11, R04B5.5, } \\
\text { F18E3.7, F59B8.2, ZC443.1, F13D12.4, ZK829.4, K12G11.3, K09D9.2, C30G12.2, Y5H2B.5, } \\
\text { F01F1.6, C49G7.8 }\end{array}$ \\
\hline Carbohydrate metabolic process & 0.0147 & $\begin{array}{l}\text { R09B5.6, C05E4.9, Y22F5A.4, Y22F5A.5, T19H12.9, F07A11.2, F07A11.5, R11F4.1, C02A12.4, } \\
\text { H23N18.1, Y37E11AR.5, T12B3.3, Y73F4A.2, ZK678.8, F01F1.12, R11D1.11, R05F9.6, T19H12.10, } \\
\text { D2096.3, H22K11.2, H02112.1, C05C8.7, R09D1.10, Y46G5A.31, C33C12.3, R11A5.4 }\end{array}$ \\
\hline
\end{tabular}


Table 4 Comparison of biological processes altered in wrn-1 mutant or wild type C. elegans strains treated with vitamin C

\begin{tabular}{|c|c|c|}
\hline \multicolumn{3}{|c|}{ Down in wrn-1 mutant + vitamin C vs untreated wrn-1 mutant $C$. elegans strain } \\
\hline Category & Benjamini $P$-value & Genes \\
\hline Locomotion & 0.0081 & $\begin{array}{l}\text { K07D8.1, C29F4.1, W05B2.1, T10E10.2, T28C6.6, T10E10.6, F46B3.5, F38A3.2, F55C10.3, } \\
\text { T01B10.2, T28C6.4, C44C10.1, T22A3.8, F13H6.1, C26C6.3, F30B5.1, ZK270.1, Y4C6B.2, } \\
\text { ZK678.5, W05B2.6, T07H6.3, K07C10.1, W05B2.5, Y41E3.2 }\end{array}$ \\
\hline Anatomical structure development & 0.0065 & $\begin{array}{l}\text { C29F4.1, T10E10.2, T10E10.6, C29E4.1, C09G5.4, F38A3.2, T01B10.2, C44C10.1, } \\
\text { F37B4.2, T07H6.3, F52B11.4, W05B2.1, T22B3.1, F27C1.8, T28C6.6, F55C10.3, } \\
\text { T28C6.4, T22A3.8, F30B5.1, F11G11.10, B0491.2, F11G11.12, ZK270.1, W05B2.6, } \\
\text { Y41E3.2, W05B2.5 }\end{array}$ \\
\hline
\end{tabular}

Up in wrn-1 mutant + vitamin C vs untreated wrn-1 mutant C. elegans strain

No significant category

\begin{tabular}{lcl}
\hline \begin{tabular}{l} 
Down in N2 + vitamin C vs untreated N2 C. elegans strain \\
\hline Category
\end{tabular} & \multicolumn{1}{l}{ Benjamini P-value } & Genes \\
\hline Proteolysis & 0.0408 & T23F4.4, ZK1037.10, F53A9.2, K07B1.1, W07B8.4, T19D2.1, W01A8.6, \\
& & C29F3.2, B0222.4, F47H4.10, F42A10.8, Y95B8A.1, R10H1.5, C07D10.4
\end{tabular}

Up in N2 + vitamin C vs untreated N2 C. elegans strain

No significant category

Finally, the list of genes in the vitamin $C$ treated wild type versus untreated wild type worms was very different from the list of differentially expressed genes in untreated wrn-1 (gk99) versus vitamin C treated wrn-1 (gk99) worms. Only four genes were regulated in a similar way in both lists and they included mup-4, C27D9.2 (down regulated), F12A10.1, and ugt-18 (up regulated). The mup-4 gene encodes a transmembrane protein required for junctional attachments between hypodermis and muscle [26]. The ugt-18 gene encodes a protein with putative UDP- glucuronosyltransferase activities (referenced in www.ncbi.nlm.nih.gov/gene/179759). The C27D9.2 and F12A10.1 genes code for uncharacterized proteins (www. wormbase.org). These results indicate that although vitamin $C$ increased the life span of both mutant and wild type worms, it affected different sets of genes and biological processes in both strains.

The life span of $w r n-1$ ( $g k 99)$ worms treated with vitamin $\mathrm{C}$ is similar to the life span of untreated wild type worms (Figure 1). We therefore compared the global expression of vitamin C treated wrn-1 $(g k 99)$ worms with the global expression of untreated wild type worms. There were 375 down regulated and 181 up regulated genes in the vitamin C treated wrn-1 $(g k 99)$ worms compared to untreated wild type worms, respectively (Additional file 6: Table S6). Categorization of the genes in our list using DAVID revealed three significantly up regulated biological processes (Benjamini $P$-value $<0.05$ ) in the vitamin $C$ treated wrn-1 ( $g$ k99) worms compared to untreated wild type worms (Table 5). Major biological processes that were up regulated in vitamin C wrn-1 ( $\mathrm{k}$ k99) mutant worms included lipid, cellular ketone, and organic acid metabolic processes (Table 5).
We next examined the genes that were commonly altered in untreated and vitamin C treated wrn-1 ( $k$ k99) worms taking the global expression of the wild type strain as a reference. The Venn diagrams in Figure 3 indicate on one hand that the expression of 472 genes from the $w r n-1(g k 99)$ worms were changed similarly in the absence or presence of vitamin $\mathrm{C}$ when compared to wild type worms. There were 317 and 155 genes down and up regulated, respectively, in the wrn-1 $(g k 99)$ worms with or without vitamin C compared to untreated wild type worms. The list of these genes can be found in (Additional file 7: Table S7). DAVID analyses on the commonly up and down regulated genes indicated that the wrn-1 $(g k 99)$ mutation increased the expression of genes involved in oxidation-reduction, cellular ketone, organic acid, and lipid metabolic processes (Table 6). These results indicate that vitamin $C$ did not revert the expression of genes involved in these biological processes in the wrn-1 (gk99) worms.

\section{Potential transcription factors involved in vitamin \\ C response in wrn-1(gk99) and wild type C. elegans}

We next performed a transcription factors binding site enrichment analysis on the promoters of the genes altered by vitamin $C$ in both wrn-1 $(g k 99)$ and wild type strains. We used publically available ChIP-seq data recently generated for 164 transcription factors in C. elegans [27]. Promoter analysis of all the genes differentially expressed by vitamin $\mathrm{C}$ in wild type worms revealed a significant enrichment of binding sites for NHR-28 and MAB-5 transcription factors (see the Additional file 8: Table S8). The C. elegans NHR-28 protein is an orphan nuclear hormone receptor with zinc finger domains required for DNA binding (www.wormbase.org). MAB-5 is a homeodomain transcription factor 
Table 5 Biological processes altered in vitamin C treated wrn-1 mutant compared to the untreated wild type C. elegans strain

\begin{tabular}{lcl}
\hline Up in wrn-1 mutant + vitamin C vs untreated N2 C. elegans strain \\
\hline Category & Benjamini $\boldsymbol{P}$-value & Genes \\
\hline Lipid metabolic process & 0.0014 & R09B5.6, F35C11.5, F08A8.2, F01D4.2, K11D2.2, F09C8.1, C48B4.1, \\
& & ZC416.6, Y49E10.18, ZC443.5, T05E7.1, F46G10.4, F01G10.3, C40H1.7 \\
Cellular ketone metabolic process & 0.0039 & C44E4.3, R09B5.6, ZK829.4, Y73F4A.2, T21F4.1, F08A8.2, R08E5.2, \\
& & M02D8.4, F01G10.3, C48B4.1, ZC416.6 \\
Organic acid metabolic process & 0.0043 & C44E4.3, R09B5.6, ZK829.4, Y73F4A.2, T21F4.1, F08A8.2, \\
& & R08E5.2, M02D8.4, F01G10.3, C48B4.1, ZC416.6 \\
\hline
\end{tabular}

related to the Antennapedia and Ultrabithorax family of homeodomain proteins. It regulates proliferation, differentiation and morphogenesis in C. elegans [28]. The expression of $n h r-28$ and mab-5 genes was not significantly changed in vitamin $C$ treated compared to untreated wild type animals (Additional file 1: Table S1). These results suggest that vitamin $\mathrm{C}$ altered the activity of these transcription factors at a post-transcriptional level in wild type animals.

Promoter analysis of all the genes differentially expressed by vitamin $\mathrm{C}$ in wrn-1(gk99) mutant worms revealed a significant enrichment of binding sites for TLP-1, NHR76, PHA-4 and NHR-28 transcription factors (see the Additional file 9: Table S9). TLP-1 is a zinc finger protein of the Sp family of transcription factor. It is a transcription factor that acts downstream of Wnt signals and that control cell polarity and patterning in C. elegans [29]. NHR-76 is a nuclear hormone receptor transcription factor involved in body fat regulation in C. elegans [30]. PHA-4 is a transcription factor of the FoxA (forkhead) family that mediates diet-restriction induced longevity in C. elegans [31]. It also antagonizes the Target of Rapamycin pathway in C. elegans [32]. The expression level of these four transcription factors in wrn-1 $(g k 99)$ mutant worms was not significantly changed by the addition of vitamin $\mathrm{C}$ (Additional file 1: Table S1). These results suggest that vitamin $C$ altered the activity of these transcription factors at a post-transcriptional level in wrn-1(gk99) mutant worms.

\section{Discussion}

We have previously observed that homozygous mice lacking exons encoding part of the helicase domain of the Werner syndrome gene product have a reduced mean life span of $16.5 \%$ compared to wild type mouse [16].

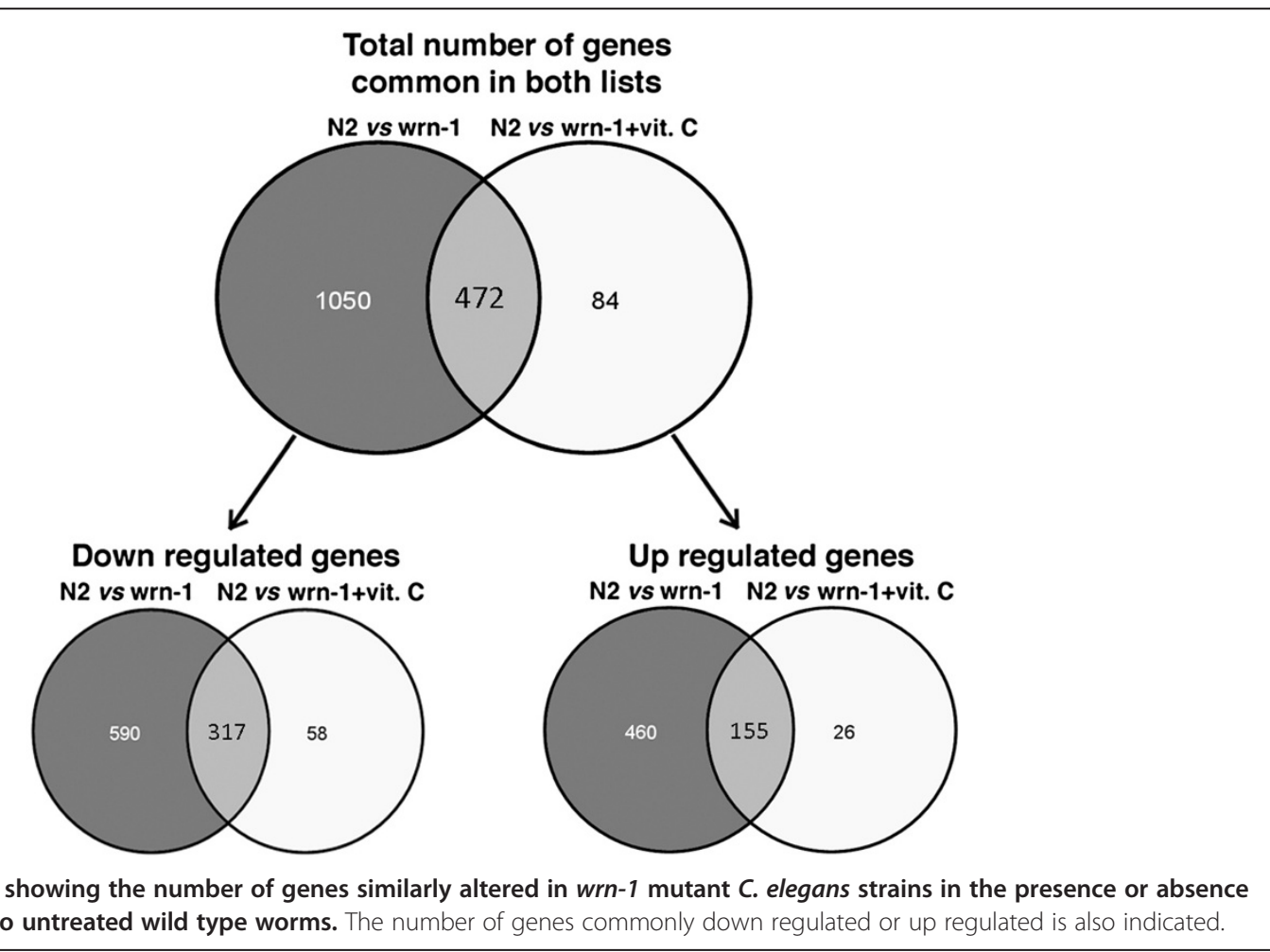


Table 6 Biological processes commonly altered in the wrn-1 mutant C. elegans strain in the presence or absence of vitamin C

\begin{tabular}{|c|c|c|}
\hline \multicolumn{3}{|c|}{ Up in wrn-1 mutant+/-vitamin C vs untreated N2 C. elegans strain } \\
\hline Category & Benjamini $P$-value & Genes \\
\hline Cellular ketone metabolic process & 0.0015 & $\begin{array}{l}\text { C44E4.3, R09B5.6, ZK829.4, Y73F4A.2, T21F4.1, F08A8.2, R08E5.2, } \\
\text { M02D8.4, F01G10.3, C48B4.1, ZC416.6 }\end{array}$ \\
\hline Organic acid metabolic process & 0.0022 & $\begin{array}{l}\text { C44E4.3, R09B5.6, ZK829.4, Y73F4A.2, T21F4.1, F08A8.2, R08E5.2, M02D8.4, } \\
\text { F01G10.3, C48B4.1, ZC416.6 }\end{array}$ \\
\hline Lipid metabolic process & 0.0028 & $\begin{array}{l}\text { Y49E10.18, R09B5.6, F35C11.5, F08A8.2, K11D2.2, T05E7.1, F46G10.4, } \\
\text { F01G10.3, C48B4.1, F09C8.1, C40H1.7, ZC416.6 }\end{array}$ \\
\hline Oxidation-reduction & 0.0391 & $\begin{array}{l}\text { K08C7.5, R07B7.5, R02D3.1, ZK829.4, K12G11.3, C30G12.2, } \\
\text { K09D9.2, T13C5.1, F08A8.2, R04B5.5, C48B4.1 }\end{array}$ \\
\hline
\end{tabular}

Importantly, such mutant mice exhibit an abnormally high oxidative stress that can be neutralized by supplementation of vitamin $C$ in drinking water [16]. In fact, vitamin $\mathrm{C}$ re-established the normal mean life span of Wrn helicase mutant mice. Interestingly, C. elegans lacking the wrn-1 helicase ortholog also have a reduced mean life span compared to wild type worms [19]. The global mRNA expression of whole wrn-1(gk99) worms by RNA-seq analyses indicated major changes in set of genes involved in overall carbon metabolism (sugars and lipids). These results are consistent with the premature aging phenotype observed in worms with a reduced WRN-1 helicase activity $[19,33]$. Although the analysis was performed on mRNA from L4 stage C. elegans, altered expression of these genes will have an effect on the duration of adulthood. Indeed, alterations in reactive oxygen species and ATP levels has been observed in adult wrn-1 (gk99) mutant worms [19]. Notably, alteration in the expression of several sets of genes involved in growth and lipid or fatty acid metabolism has not only been observed in cultured Wrn mutant mouse embryonic fibroblasts but also in the liver of $\mathrm{Wrn}$ helicase mutant mice [16,34]. Importantly, since we noticed a three hours developmental delay in wrn-1 ( $k$ k99) animals compared to wild type, we carefully monitored physiological developmental cues (i.e. vulva and gonad development) in both animal population before harvesting them to perform mRNA analysis (as described in the Methods). Therefore, the change of the expression profile of genes involved in different biochemical pathways observed between wrn-1(gk99) mutant and wild type animals reflect accurately the difference detected at a specific developmental time point rather than a developmental shift caused by the loss of wrn-1. Detailed comparative microscopic and genetic analyses along with global mRNA expression analyses at several developmental stages will be necessary to acquire a better picture of changes as a function of not only chronological but also biological age in wrn-1(gk99) mutant animals.
Vitamin $C$ treatment increased the mean life span of wrn-1 ( $k$ k99) worms to untreated wild type levels. This is similar to the result obtained with mice. Vitamin $C$ supplementation rescued the shorter mean life span of $\mathrm{Wrn}$ mutant mice [16]. Vitamin $C$ also significantly increased the life span of wild type C. elegans (Figure 1). We did not see an increase in the life span of wild type mice supplemented with vitamin $C$ in a previous study [16]. Unlike mice, wild type $C$. elegans do not have the enzyme required for the synthesis of their own vitamin $C$ as far as we can tell. However, C. elegans contains three potential cytochromes b561 encoding genes (F55H2.5, F39G3.4 and F39G3.5) [35]. Cytochromes b561 are intrinsic membrane proteins containing two heme molecules, and reducible by ascorbate. They have been suggested to function as electron transporters, shuttling electrons across membranes from ascorbate to an acceptor molecule. The one-electron oxidation product of ascorbate, monodehydro-ascorbate has been shown, at least in vitro, to function as an electron acceptor for cytochromes b561. This reaction results in the regeneration of a fully reduced ascorbate molecule [35]. The presence of Cytochromes b561 encoding genes in $C$. elegans suggests that this species could potentially use vitamin $C$ in different chemical reactions [36]. The impact of vitamin $C$ on the levels of different metabolites required for energy or impacting on ROS levels in the body of wild type and wrn-1(gk99) C. elegans is currently under investigation. Interestingly, the expression of the F39G3.4 gene is reduced in wrn-1(gk99) mutants (Additional file 3: Table S3) but was not changed by the addition of vitamin C (Additional file 4: Table S4). The F39G3.4 gene may be required for the regeneration of ascorbate from monodehydro-ascorbate in wrn-1 ( $k$ k99) mutants, a hypothesis that will require confirmation.

Our results indicate a major difference in gene expression response to vitamin $C$ between wild type and mutant worms. The list of differentially expressed genes in untreated wrn-1 (gk99) mutants versus vitamin C treated wrn$1(g k 99)$ mutants was very different from the list of genes in vitamin $C$ treated wild type versus untreated wild type 
worms (Additional file 4: Tables S4 and Additional file 5: Table S5). These results indicate that although vitamin $C$ increased the life span of both mutant and wild type worms, it affected different sets of genes in both strains. Another important difference is that the mean life span of vitamin C treated wrn-1 ( $\mathrm{kk} 99)$ worms is shorter ( $\sim$ days) than vitamin $C$ treated wild type worms (11 days) (Figure 1). The median life span of wrn-1 (gk99) worms treated with vitamin $C$ was close to untreated wild type worms. The expression profile of vitamin C treated wrn- 1 ( $g k 99)$ worms compared to untreated wrn-1 (gk99) worms revealed down regulation of genes involved in biological processes related to locomotion and anatomical structure development. Upon examination of the global mRNA expression analysis of treated and untreated wrn-1 (gk99) worms (taking the global mRNA expression of wild type as a reference), we found that the expression of 472 genes at the mRNA level was similarly altered in mutant worms compared to wild type worms in the presence or absence of vitamin C. The expression of these genes was affected by the wrn-1 ( $g$ k99) mutation but was not reversed by vitamin C suggesting that alteration in expression of these genes is not required for the impact of vitamin $C$ on $C$. elegans life span. These genes are involved in oxidation-reduction, cellular ketone, organic acid, and lipid metabolic processes (Table 6). Thus, vitamin C extended the life span of wrn-1 ( $g k 99)$ worms by altering biological processes not only involved in locomotion but also in anatomical structure development (Table 4). Such processes are indeed affected at the physiological levels, as a knock down of WRN-1 protein expression by the RNA interference technique results in various developmental defects, including small, dumpy, ruptured, transparent body, growth arrest and bag of worms [33].

The mechanism by which vitamin $\mathrm{C}$ alters gene expression in mutant and wild type C. elegans is unknown. Our analysis of enrichment for specific transcription factor binding sites in the altered genes in the presence of vitamin $C$ have indicated changes in the activity of hormone receptor-type transcription factors (like NHR-28 and NHR-76) and of PHA-4, a transcription factors which activity depends on pathways affecting the status of several metabolites and/or nutrients in C. elegans. One important pathway modulated by metabolites is the CeTOR pathway that affects PHA-4 activity [32]. Our hypothesis is that vitamin C not only affects the redox status of cell in C. elegans, but also important metabolites that may be used as ligands for nuclear receptors (lipid derived steroidogenic metabolites) or for signaling molecules that regulate the activity of different transcription factors. Metabolomics analysis with both wrn-1(gk99) mutant and the N2 wild type strains in the absence or presence of vitamin $\mathrm{C}$ is underway and is the scope of another study.

\section{Conclusions}

To conclude, our study indicates that $10 \mathrm{mM}$ vitamin $\mathrm{C}$ in the growth media increases the mean life span of the wrn-1(gk99) mutant and the N2 wild type strains at $25^{\circ}$ C. However, the alteration of gene expression by vitamin $\mathrm{C}$ is different between wrn-1 $(\mathrm{gk} 99)$ and wild type strains. Based on our criteria to generate lists of differentially expressed genes between genotypes, we observed alteration in the expression of 1522 genes in wrn-1 ( $k k 99)$ worms compared to wild type worms. Vitamin C did not significantly modify the alteration of 472 of these genes. Such genes are mainly involved in oxidation-reduction, cellular ketone, organic acid, carbohydrate, and lipid, metabolic processes. Vitamin $\mathrm{C}$, in return, altered the expression of several genes in wrn-1(gk99) worms involved in locomotion and anatomical structure development. It is interesting to mention that vitamin $\mathrm{C}$ can rescue the morphology of liver and adipose tissues of Wrn helicase mutant mice [16]. This report thus provides lists of genes that can potentially affect the healthy aging of not only wild type but also wrn-1 ( gk99) worms upon genetic manipulation in the presence of vitamin C. It also provides important clues on the biological processes that vitamin $\mathrm{C}$ affects to increase life span in $C$. elegans.

\section{Methods}

\section{Caenorhabditis elegans strains}

All C. elegans strains were maintained as described [37]. The wrn-1 ( $k k 99)$ strain was obtained from the C. elegans Genetics Center (University of Minnesota, St Paul, MN) and was out-crossed four times with the wild type N2 strain to remove possible unrelated mutations. The wrn-1 ( $g k 99$ ) contains a 196 bps deletion that inhibits the expression of the protein [38]. The primers used to genotype this strain are 5'-CTGGCTGTAACTGCACCTGA-3' and 5'-AAATGGGAGGGAAAGAGCAT-3'. To measure life span, worms were transferred to fresh plates and were grown at $25^{\circ} \mathrm{C}$. Death was scored by absence of any movement after several light pokes with a platinum wire.

\section{Extraction of total RNA and sequencing}

Eggs were isolated by standard bleach treatment and hatched overnight at room temperature. The resulting L1 larvae were then distributed over NGM plates supplemented or not with $10 \mathrm{mM}$ vitamin C (Sodium Lascorbate) (Sigma-Aldrich, ST-Louis, MO) and grown at $25^{\circ} \mathrm{C}$. A concentration of $10 \mathrm{mM}$ ascorbate was chosen in this study as it has previously been shown that such a concentration did not influence the survival of C. elegans [25]. The developmental stage of the animals was monitored visually until the worms reached the larvae L4 stage. Animals were precisely staged by observing vulval and gonad formation by Nomarski 
optics before collecting the animals for analysis as described previously [39]. Worms were spun down in an eppendorf tube and lysed in TRIZOL (Invitrogen, Carlsbad, CA) to extract total RNA. RNA quality was checked with an Agilent Bioanalyzer 2100 and libraries were made using the Illumina's TruSeq v2 kit according to the manufacturer's recommendations. The RIN (RNA Integrity Number) of all samples was above 8.0. Fifty base pairs paired end sequencing was performed on the HiSeq 2000 machine form Illumina.

\section{RNA-seq analyses}

The data discussed in this publication have been deposited in NCBI's Gene Expression Omnibus http://www.ncbi.nlm. nih.gov/geo/ [40] and are accessible through GEO Series accession number GSE54173. Table 1 contains the read statistics for the raw reads coming off the sequencer. Detailed statistics on the quality of the reads were calculated with FastQC (http://www.bioinformatics.babraham.ac. $\mathrm{uk} /$ projects/fastqc/). The 50 base pairs raw sequences were aligned on the C. elegans ce10/W220 genome with TopHat [41] using the Ensembl annotations provided with the Illumina iGenomes. The htseq-count software (http://www-huber.embl.de/users/anders/HTSeq) was used to count the number of reads aligned to each gene. These counts were then normalized relative to the sequencing depth with DESeq [42]. DESeq was also used to calculate the fold changes, log fold changes, and significance of the changes for each comparison.

Enrichments for specific biological functions using the DAVID (Database for Annotation, Visualization, and Integrated Discovery) web site [43] were considered significant with a $P$-value smaller than 0.05 .

\section{Validation of mRNA expression}

The quantitative measure of selected mRNA expressions was performed by qRT-PCR. Worms were spun down in an eppendorf tube and lysed in TRIZOL (Invitrogen, Carlsbad, CA) to extract total RNA. PolyA + RNA was isolated with the RNeasy plus mini kit (Qiagen, Mississauga, $\mathrm{ON}$ ). The cDNAs were obtained by reverse transcription using the specific 3'-primer on total RNA (Additional file 2: Table S2 for primer design). cDNA were then subjected to $\mathrm{qPCR}$ reaction using PerfeCTa ${ }^{\circ} \mathrm{SYBR}^{\circ}$ Green FastMix ${ }^{\circ}$, Low ROX $^{\mathrm{mm}}$ (Quanta Bioscience, Gaithersburg, MD) according to manufacturer instruction. At the end of each run, samples were analyzed on a $2 \%$ agarose gel to determine the quality of the amplification. Primers used for qRT-PCR are listed in (Additional file 2: Table S2).

\section{modENCODE transcription factors binding site} enrichment analysis

We downloaded a list of 164 significant ChIP-seq peaks mapped on the ws220 C. elegans genome from the
modENCODE FTP website (ftp://data.modencode.org/) [27]. Using this significant list of peaks, we first defined for every transcription factors a list of genes located within $500 \mathrm{bp}$ of at least one significant peak. Using this list of genes we computed the overlap enrichment scores between the list of genes targeted by the transcriptions factors and the list of significantly differentially expressed genes identified in our wrn-1 and wild type gene expression experiments for up-regulated, down-regulated and all significant genes. The enrichment was evaluated based on a one-sided hypergeometric distribution. We consider an enrichment between genes targeted by a transcription factor and the list of genes modulated by vitamin $\mathrm{C}$ to be significant if the Benjamini-Hochberg adjusted p-value is lower than 0.05 .

\section{Additional files}

Additional file 1: Table S1. Normalized counts for each biological replicate. This list gives the normalized number of reads for each gene in each replicate.

Additional file 2: Table S2. Primers used for the quantitative RT-PCR.

Additional file 3: Table S3. List of genes found to be differentially expressed in the wrn-1 mutant compared to the wild type N2 worms. The columns labeled baseMeanA and baseMeanB represent the mean number of reads (from the normalized counts of Additional file 1: Table S1) for the N2 wild type and the wrn-1(gk99) strains, respectively.

Additional file 4: Table S4. List of genes found to be differentially expressed in the vitamin $C$ treated wrn-1 mutant compared to untreated wrn-1 mutant worms. The columns labeled baseMeanA and baseMeanB represent the mean number of reads (from the normalized counts of Additional file 1: Table S1) for the untreated wrn-1(gk99) and the vitamin C treated wrn-1 ( $\mathrm{gk99}$ ) worms, respectively.

Additional file 5: Table S5. List of genes found to be differentially expressed in the vitamin C treated wild type N2 compared to untreated wild type N2 worms. The columns labeled baseMeanA and baseMeanB represent the mean number of reads (from the normalized counts of Additional file 1: Table S1) for the untreated N2 wild type and the vitamin C treated N2 wild type worms, respectively.

Additional file 6: Table S6. List of genes found to be differentially expressed in the vitamin $C$ treated wrn-1 mutant compared to untreated wild type worms. The columns labeled baseMeanA and baseMeanB represent the mean number of reads (from the normalized counts of Additional file 1: Table S1) for the untreated N2 wild type and the vitamin C treated wrn-1 ( $\mathrm{gk99}$ ) worms, respectively.

Additional file 7: Table S7. Genes differentially expressed in a similar way in wrn-1 mutant worms incubated with or without vitamin C compared to the untreated wild type N2 worms.

Additional file 8: Table S8. List of transcription factors potentially binding to the promoters of genes altered by vitamin $\mathrm{C}$ in wild type worms.

Additional file 9: Table S9. List of transcription factors potentially binding to the promoter of genes altered by vitamin C in wrn-1 mutant worms.

\section{Competing interests}

The authors declare that they have no competing interests.

\section{Authors' contributions}

AD maintained the worms in the laboratory of MJS and performed the life span studies. SP extracted the total RNA from each treated and untreated worm cultures and validated the expression of several genes by qRT-PCR. ML wrote the manuscript. All authors approved the final submission. 


\section{Acknowledgments}

We thank Dr. Odile Neyret-Djossou from the sequencing platform and Alexis Blanchet-Cohen from the bioinformatics platform at the Institut de Recherche Clinique de Montréal (Québec, Canada) for their valuable help. We thank E.R. Paquet from the Centre de Recherche sur le Cancer de l'Université Laval (Quebec City) for the transcription factor analyses. Nematode strains were provided by the Caenorhabditis Genetics Center, which is funded by the National Institute of Health National Center for Research Resources. This work was supported by the Canadian Institutes of Health Research to M.L. and M.JS. M.J.S. is a Senior scholar from the Fonds de la Recherche du Québec-Santé.

Received: 7 April 2014 Accepted: 15 October 2014

Published: 27 October 2014

\section{References}

1. Epstein CJ, Martin GM, Schultz AL, Motulsky AG: Werner's syndrome a review of its symptomatology, natural history, pathologic features, genetics and relationship to the natural aging process. Medicine (Baltimore) 1966, 45(3):177-221.

2. Salk D: Werner's syndrome: a review of recent research with an analysis of connective tissue metabolism, growth control of cultured cells, and chromosomal aberrations. Hum Genet 1982, 62(1):1-5.

3. Yu CE, Oshima J, Fu YH, Wijsman EM, Hisama F, Alisch R, Matthews S, Nakura J, Miki T, Ouais S, Martin GM, Mulligan J, Schellenberg GD: Positional cloning of the Werner's syndrome gene. Science 1996, 272(5259):258-262.

4. Ozgenc A, Loeb LA: Current advances in unraveling the function of the Werner syndrome protein. Mutat Res 2005, 577(1-2):237-251.

5. Kamath-Loeb AS, Shen JC, Loeb LA, Fry M: Werner syndrome protein. II. Characterization of the integral 3' - > 5' DNA exonuclease. J Biol Chem 1998, 273(51):34145-34150.

6. Huang S, Beresten S, Li B, Oshima J, Ellis NA, Campisi J: Characterization of the human and mouse WRN 3'- > 5' exonuclease. Nucleic Acids Res 2000, 28(12):2396-2405.

7. Balajee AS, Machwe A, May A, Gray MD, Oshima J, Martin GM, Nehlin JO, Brosh R, Orren DK, Bohr VA: The Werner syndrome protein is involved in RNA polymerase II transcription. Mol Biol Cell 1999, 10(8):2655-2668.

8. Cooper MP, Machwe A, Orren DK, Brosh RM, Ramsden D, Bohr VA: Ku complex interacts with and stimulates the Werner protein. Genes Dev 2000, 14(8):907-912.

9. Shen JC, Loeb LA: The Werner syndrome gene: the molecular basis of RecQ helicase-deficiency diseases. Trends Genet 2000, 16(5):213-220.

10. Saintigny Y, Makienko K, Swanson C, Emond MJ, Monnat RJ Jr: Homologous recombination resolution defect in werner syndrome. Mol Cell Biol 2002, 22(20):6971-6978.

11. Crabbe L, Verdun RE, Haggblom Cl, Karlseder J: Defective telomere lagging strand synthesis in cells lacking WRN helicase activity. Science 2004, 306(5703):1951-1953.

12. Lebel M, Leder P: A deletion within the murine Werner syndrome helicase induces sensitivity to inhibitors of topoisomerase and loss of cellular proliferative capacity. Proc Natl Acad Sci U S A 1998, 95(22):13097-13102.

13. Lebel M, Cardiff RD, Leder P: Tumorigenic effect of nonfunctional p53 or p21 in mice mutant in the Werner syndrome helicase. Cancer Res 2001, 61(5):1816-1819

14. Massip L, Garand C, Turaga RV, Deschenes F, Thorin E, Lebel M: Increased insulin, triglycerides, reactive oxygen species, and cardiac fibrosis in mice with a mutation in the helicase domain of the Werner syndrome gene homologue. Exp Gerontol 2006, 41(2):157-168.

15. Lebel M, Lavoie J, Gaudreault I, Bronsard M, Drouin R: Genetic cooperation between the Werner syndrome protein and poly(ADP-ribose) polymerase-1 in preventing chromatid breaks, complex chromosomal rearrangements, and cancer in mice. Am J Pathol 2003, 162(5):1559-1569.

16. Massip L, Garand C, Paquet ER, Cogger VC, O'Reilly JN, Tworek L, Hatherell A, Taylor CG, Thorin E, Zahradka P, Le Couteur DG, Lebel M: Vitamin C restores healthy aging in a mouse model for Werner syndrome. FASEB J 2010, 24(1):158-172.

17. Lee JS, Chu IS, Mikaelyan A, Calvisi DF, Heo J, Reddy JK, Thorgeirsson SS: Application of comparative functional genomics to identify best-fit mouse models to study human cancer. Nat Genet 2004, 36(12):1306-1311.
18. Hyun M, Bohr VA, Ahn B: Biochemical characterization of the WRN-1 RecQ helicase of Caenorhabditis elegans. Biochemistry 2008, 47(28):7583-7593.

19. Dallaire A, Garand C, Paquel ER, Mitchell SJ, de Cabo R, Simard MJ, Lebel M: Down regulation of miR-124 in both Werner syndrome DNA helicase mutant mice and mutant Caenorhabditis elegans wrn-1 reveals the importance of this microRNA in accelerated aging. Aging 2012, 4(9):636-647.

20. Mortazavi A, Williams BA, McCue K, Schaeffer L, Wold B: Mapping and quantifying mammalian transcriptomes by RNA-Seq. Nat Methods 2008, 5(7):621-628.

21. Wang Z, Gerstein M, Snyder M: RNA-Seq: a revolutionary tool for transcriptomics. Nat Rev Genet 2009, 10(1):57-63.

22. Marioni JC, Mason CE, Mane SM, Stephens M, Gilad Y: RNA-seq: an assessment of technical reproducibility and comparison with gene expression arrays. Genome Res 2008, 18(9):1509-1517.

23. Hawkins RD, Hon GC, Ren B: Next-generation genomics: an integrative approach. Nat Rev Genet 2010, 11(7):476-486.

24. Hallegger M, Llorian M, Smith CW: Alternative splicing: global insights. FEBS J 2010, 277(4):856-866.

25. Huang J, Lemire BD: Mutations in the C. elegans succinate dehydrogenase iron-sulfur subunit promote superoxide generation and premature aging. J Mol Biol 2009, 387(3):559-569.

26. Gatewood BK, Bucher EA: The mup-4 locus in Caenorhabditis elegans is essential for hypodermal integrity, organismal morphogenesis and embryonic body wall muscle position. Genetics 1997, 146(1):165-183.

27. Niu W, Lu ZJ, Zhong M, Sarov M, Murray JI, Brdlik CM, Janette J, Chen C, Alves P, Preston E, Slightham C, Jiang L, Hyman AA, Kim SK, Waterston RH, Gerstein M, Snyder M, Reinke V: Diverse transcription factor binding features revealed by genome-wide ChIP-seq in C. elegans. Genome Res 2011, 21(2):245-254.

28. Salser SJ, Kenyon C: A C. elegans Hox gene switches on, off, on and off again to regulate proliferation, differentiation and morphogenesis. Development 1996, 122(5):1651-1661.

29. Zhao X, Yang Y, Fitch DH, Herman MA: TLP-1 is an asymmetric cell fate determinant that responds to Wnt signals and controls male tail tip morphogenesis in C. elegans. Development 2002, 129(6):1497-1508.

30. Noble T, Stieglitz J, Srinivasan S: An integrated serotonin and octopamine neuronal circuit directs the release of an endocrine signal to control $C$. elegans body fat. Cell Metab 2013, 18(5):672-684.

31. Panowski SH, Wolff S, Aguilaniu H, Durieux J, Dillin A: PHA-4/Foxa mediates diet-restriction-induced longevity of C. elegans. Nature 2007, 447(7144):550-555.

32. Sheaffer KL, Updike DL, Mango SE: The Target of Rapamycin pathway antagonizes pha-4/FoxA to control development and aging. Current Biology : CB 2008, 18(18):1355-1364.

33. Lee SJ, Yook JS, Han SM, Koo HS: A Werner syndrome protein homolog affects $C$. elegans development, growth rate, life span and sensitivity to DNA damage by acting at a DNA damage checkpoint. Development 2004, 131(11):2565-2575.

34. Turaga RV, Paquet ER, Sild M, Vignard J, Garand C, Johnson FB, Masson $J Y$, Lebel M: The Werner syndrome protein affects the expression of genes involved in adipogenesis and inflammation in addition to cell cycle and DNA damage responses. Cell Cycle 2009, 8(13):2080-2092.

35. Verelst W, Asard H: A phylogenetic study of cytochrome b561 proteins. Genome Biol 2003, 4(6):R38

36. Myllyharju J: Prolyl 4-hydroxylases, key enzymes in the synthesis of collagens and regulation of the response to hypoxia, and their roles as treatment targets. Ann Med 2008, 40(6):402-417.

37. Brenner S: The genetics of Caenorhabditis elegans. Genetics 1974, 77(1):71-94.

38. Bates DJ, Li N, Liang R, Sarojini H, An J, Masternak MM, Bartke A, Wang E: MicroRNA regulation in Ames dwarf mouse liver may contribute to delayed aging. Aging Cell 2010, 9(1):1-18.

39. Bosse GD, Ruegger S, Ow MC, Vasquez-Rifo A, Rondeau EL, Ambros VR Grosshans H, Simard MJ: The decapping scavenger enzyme DCS-1 controls microRNA levels in Caenorhabditis elegans. Mol Cell 2013, 50(2):281-287

40. Edgar R, Domrachev M, Lash AE: Gene expression omnibus: NCBI gene expression and hybridization array data repository. Nucleic Acids Res 2002, 30(1):207-210. 
41. Trapnell C, Roberts A, Goff L, Pertea G, Kim D, Kelley DR, Pimentel H, Salzberg SL, Rinn JL, Pachter L: Differential gene and transcript expression analysis of RNA-seq experiments with TopHat and Cufflinks. Nat Protoc 2012, 7(3):562-578

42. Anders $\mathrm{S}$, Reyes $\mathrm{A}$, Huber W: Detecting differential usage of exons from RNA-seq data. Genome Res 2012, 22(10):2008-2017.

43. Dennis G Jr, Sherman BT, Hosack DA, Yang J, Gao W, Lane HC, Lempicki RA: DAVID: database for annotation, visualization, and integrated discovery. Genome Biol 2003, 4(5):3.

doi:10.1186/1471-2164-15-940

Cite this article as: Dallaire et al:: Expression profile of Caenorhabditis elegans mutant for the Werner syndrome gene ortholog reveals the impact of vitamin C on development to increase life span. BMC

Genomics 2014 15:940.

\section{Submit your next manuscript to BioMed Central and take full advantage of:}

- Convenient online submission

- Thorough peer review

- No space constraints or color figure charges

- Immediate publication on acceptance

- Inclusion in PubMed, CAS, Scopus and Google Scholar

- Research which is freely available for redistribution 